\title{
A High Efficiency Spread Spectrum Scheme Using Approximate Orthogonal Complex Sequences
}

\author{
Xiaohong SHI \\ School of Information Science and Engineering, Southeast University, Nanjing, China \\ E-mail: sxh@seu.edu.cn \\ Received September 15, 2009; revised October 16, 2009; accepted November 20, 2009
}

\begin{abstract}
This paper presents a high efficiency spread spectrum scheme using approximate orthogonal complex (AOC) sequences. In this scheme, the 64 AOC sequences picked up from $8^{4}$ complex sequences space are employed for spreading spectrum. In modulation, 6 input bits is used to select one AOC sequence, and the selected sequence is then phase-rotated by another 2 input bits. In demodulator, a complex correlator detects the transmitted AOC sequence. Simulation results show that the proposed scheme has better BER performance than the existing complementary code keying (CCK) modulation scheme. For AOC, additional processing gain of $1.79 \mathrm{~dB}$ can be obtained when the sequence length is 8 .
\end{abstract}

Keywords: Spread Spectrum Communications, Approximate Orthogonal Complex (AOC) Sequences, Modulation and Demodulation

\section{Introduction}

Spread spectrum communication is one of the popular ways of modern communications. It has an obvious advantage of good bit-rate-error (BER) performance, while has an abominable shortcoming of wasting valuable transmission channel bandwidth [1]. Many efforts have been done to overcome this shortcoming. Reference [2] put forward a tamed spread spectrum (TSS) method, in which several orthogonal sequences were transmitted simultaneously in one symbol. Although this method did improve transmission data rate, it increased the transmission power at the same time. This is not permitted in some applications. Complementary code keying (CCK) modulation is another efficient approach to solve the above problem. In CCK modulation [3], a group of complex complementary codes that have good co-correlative characteristics are for selection. The modulation procedure is essentially the selection of these codes. Different from the TSS method, only one complex complementary code is applied in one CCK symbol. Therefore the transmission power is not increased. CCK modulation has found wide application in WLAN and other wireless communications where high bandwidth efficiency is required.

While from the point of Modulation Efficiency (ME), CCK modulation is not the best technique. The ME can be defined as $\eta=G \cdot \frac{R_{b}}{W}$, where $R_{b}$ is the data rate,
$W$ is the bandwidth of the modulated signal, and $G$ is the processing gain related with the co-correlative coefficients (CoC) of the spreading sequences. This will be illustrated later.

Therefore increasing the processing gain $\mathrm{G}$ in receiver is an obligatory way to improve the efficiency of spreading spectrum modulation. This paper proposes a new scheme using approximate orthogonal complex (AOC) sequences for spreading spectrum. This can increase the processing gain $\mathrm{G}$ near $2 \mathrm{~dB}$. Theoretical derivation and computer simulation both give a result that the proposed AOC method has better performance than that of CCK scheme.

This paper is organized as follows. Section 2 introduces how to generate AOC sequences, and interprets co-correlative characteristic difference between AOC and CCK sequences. Section 3 proposes the AOC modulation and demodulation scheme, and section 4 gives the performance results and analysis. And in section 4, some useful conclusions are presented.

\section{AOC Sequence Generation}

Assume a complex sequence with length $L$ is $C_{m}$, where $C_{m}=\left\{c_{m}(0), c_{m}(1), \cdots, c_{m}(l), \cdots, c_{m}(L-1)\right\}$ and $c_{m}(l)=$ $c_{m}^{r}(l)+j c_{m}^{i}(l)$. Then the co-correlative value between sequence $C_{m}$ and sequence $C_{n}$ can be written as 
Table 1. The number of sequences when $L=8$.

\begin{tabular}{cc}
$q$ & number of sequences \\
\hline$=0$ & 16 \\
$\leq 2$ & 32 \\
$\leq 4$ & 64 \\
$\leq 8$ & 128 \\
\hline
\end{tabular}

$$
V_{C o C}=\sum_{l=0}^{L-1}\left[c_{m}^{r}(l) c_{n}^{r}(l)+c_{m}^{i}(l) c_{n}^{i}(l)\right]= \begin{cases}2 L, & m=n \\ q, & m \neq n\end{cases}
$$

In the total space of complex sequences with length $L$, there are $4^{L}$ complex sequences. Of these sequences, there may exist a set of sequences that has small

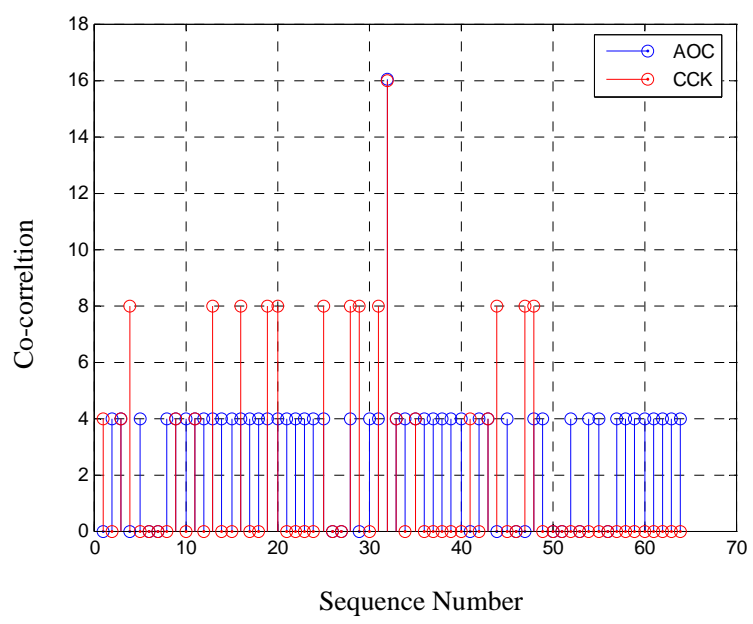

Figure 1. Co-correlation comparison of AOC with CCK sequences.

Table 2. Comparison of AOC with CCK sequences

\begin{tabular}{ccccccc}
\hline Scheme & $\begin{array}{c}\text { Number of } \\
\text { Sequences }\end{array}$ & $\begin{array}{c}\text { Length of } \\
\text { sequences }\end{array}$ & $\begin{array}{c}\text { First Peak Value of } \\
\text { Co-correlation }\end{array}$ & $\begin{array}{c}\text { Secondary Peak } \\
\text { Value of } \\
\text { Co-correlation }\end{array}$ & $\begin{array}{c}\text { Processing Gain } \\
\text { (dB) }\end{array}$ & $\begin{array}{c}\text { Modulation } \\
\text { Efficiency }\end{array}$ \\
\hline CCK & 64 & 8 & 16 & 8 & 9 & 4.5 \\
AOC & 64 & 8 & 16 & 4 & 10.79 & 5.39 \\
\hline
\end{tabular}

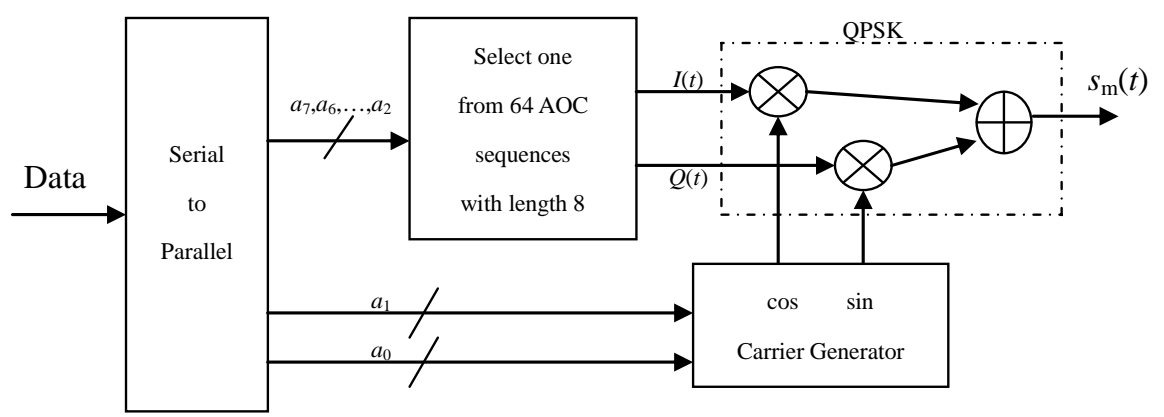

Figure 2. AOC modulator structure.

co-correlative values between any two different sequences. Generally, the bigger the value is, the larger the set is. We can search out all the sequences of the set by computer according to the predetermined $q$ parameter.

Table 1 lists the number of sequences of the set when $L$ is 8 . In this table, $q$ takes values from 0 to 8 , and the number of sequences takes values from 16 to 128 correspondingly.

From Table 1, we can obtain 64 sequences when $q \leq 4$, i.e., these 64 sequences have small co-correlative values not bigger than 4 . These selected sequences are called approximate orthogonal complex (AOC) sequences. The advantage of the AOC sequences can be taken for spreading spectrum modulation. Comparing with the existing CCK codes [3], AOC can reach higher processing gain in correlation receiver. Because AOC sequences have better co-correlation performance than CCK codes, Figure 1 shows the co-correlation comparison of AOC with CCK sequences.

In Figure 1, there are two kinds of color marked lines. The red one is co-correlation of CCK sequences, and the 


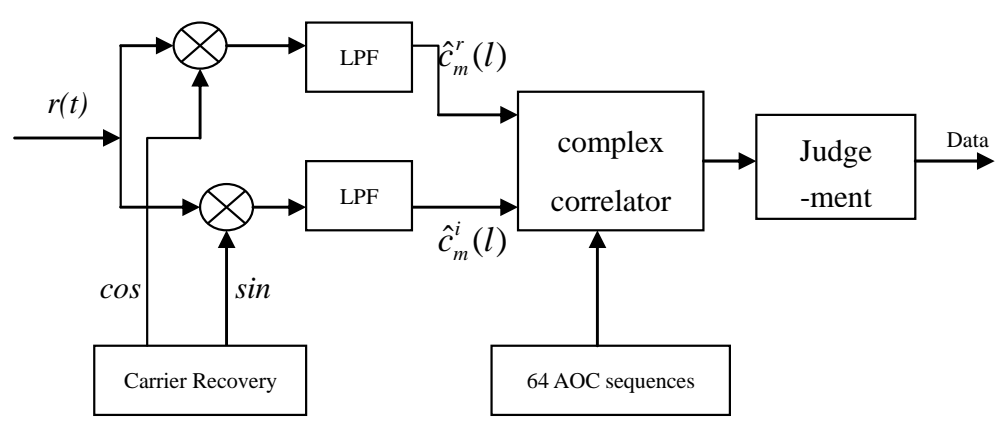

Figure 3. AOC demodulator structure.

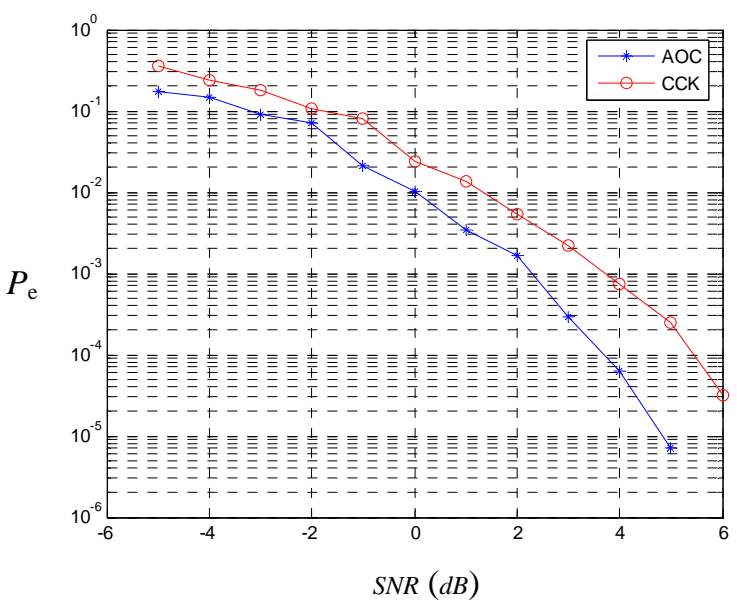

Figure 4. BER performance of AOC versus CCK.

blue one is that of AOC sequences. Co-correlation is done between any two of 64 AOC or CCK sequences. So the first peak value of the co-correlation in Figure 1 is in fact the auto-correlation value of a sequence, while the secondary peak value is really corresponding to the co-correlation between this sequence and another sequence of the said space. This figure opens out that the AOC sequences can have better co-correlation than that of CCK sequences. Because the secondary peak value of co-correlation of AOC sequences (the value is 4, marked with blue color) is much lower than that of CCK's (the value is 8 , marked with red color). This characteristic will certainly result in better processing gain in receiver with correlating operation. That is why we need AOC sequences.

Now let us give a definition of Modulation Efficiency $(M E)$. Assume the data rate of the modulator is $R_{b}$, the bandwidth of the modulated signal is $W$, and the processing gain of the demodulator due to dispread spectrum is $G$. Then the $M E$ can be defined as

$$
\eta=G \cdot \frac{R_{b}}{W}=G \cdot \frac{M}{L}
$$

where $M$ is number of bits input to the modulator, and therefore $R_{b} / \mathrm{W}$ is equal $M / L$. The factor $\eta$ reflects both the utility of channel bandwidth and the processing gain to achieve. It can express the modulation efficiency actually. In spread spectrum communications system using strict orthogonal sequences, the processing gain is exactly the length $L$ of spreading spectrum sequences, i.e., $G=L$. While for AOC and CCK applications that are not the case. Because the used sequences are not totally orthogonal to each other, and $G$ is not equal to $L$. But $G$ is essentially relevant to the gap between the first peak (i.e., the auto-correlation value) and the secondary peak of the co-correlation values and their distribution as shown in Figure 1. It is not difficult to find that the value of $G$ for CCK scheme is 8 (equals to $9 \mathrm{~dB}$ ), while that of AOC is 12 (equals to $10.79 \mathrm{~dB}$ ). That means the AOC scheme can reach additional $1.79 \mathrm{~dB}$ processing gain in principle. This result fits the performance curve in section 4 perfectly. Table 2 presents detailed comparison of AOC with CCK sequences.

\section{AOC Modulation and Demodulation}

AOC modulation employs a set of approximate orthogonal complex sequences with length $L$ for spreading spectrum. An AOC sequence defined in section 1 is composed of an in-phase sequence and a quadrature-phase sequence. They are the in-phase and the quadrature-phase inputs of QPSK modulator. A typical AOC 
modulator is shown in Figure 2.

In Figure 2, the serial input Data is converted to parallel codes. Each code has $M$ bits, and $M$ is assumed as 8 . The 6 bits named as $\left\{a_{7}, a_{6}, \ldots, a_{2}\right\}$ is used for selecting one from AOC space of $2^{6}$ sequences. The pitching on sequence is then for QPSK modulation. The 2 bits named as $\left\{a_{1}, a_{0}\right\}$ is used for rotating the QPSK carrier phase, there 4 phases of $\left\{0^{\circ}, 90^{\circ}, 180^{\circ}\right.$, and $\left.270^{\circ}\right\}$ can be added to the carrier in each AOC sequence modulation.

Refer to reference [3], the AOC modulator has a similar structure as the CCK's. The little difference between them is just in the modulation sequences. However, AOC has a more flexible mechanism in adjusting ratio of Data rate over bandwidth of modulated signal $s_{m}(t)$ by changing the $q$ value of AOC sequences. The modulated signal $s_{m}(t)$ can be described as

$$
s_{m}(t)=c_{m}^{r}(l) \cdot \cos \left[2 \pi f_{0} t+\varphi\left(a_{1}, a_{0}\right)\right]+c_{m}^{i}(l) \cdot \sin \left[2 \pi f_{0} t+\varphi\left(a_{1}, a_{0}\right)\right]
$$

where $l=0,1, \cdots, 7, f_{0}$ is the carrier frequency, and $\varphi\left(a_{1}, a_{0}\right)$ takes one value of $\left\{0^{\circ}, 90^{\circ}, 180^{\circ}\right.$, and $\left.270^{\circ}\right\}$ according to $\left\{a_{1}, a_{0}\right\}$.

AOC demodulator corresponding to Figure 2 is shown in Figure 3.

In Figure 3, the received signal is

$$
r(t)=s_{m}(t)+n(t)
$$

where $n(t)$ is assumed as additive Gausian white noise (AWGN). The complex correlator computes correlation between the down-converted complex sequence $\hat{C}_{m}=\left\{\hat{c}_{m}(0), \cdots, \hat{c}_{m}(l), \cdots, \hat{c}_{m}(L-1)\right\}$, where $\hat{c}_{m}(l)=\hat{c}_{m}^{r}(l)$ $+j \hat{c}_{m}^{i}(l)$, and one of the local AOC sequences. There are 64 complex correlation operations needed. The correlation is

$$
\begin{aligned}
& R=\sum_{l=0}^{L-1}\left[\hat{c}_{m}(l) \cdot c_{n}^{*}(l)\right] \\
& =\sum_{l=0}^{L-1}\left[\hat{C}_{m}^{r}(l) C_{n}^{r}(l)+\hat{c}_{m}^{i}(l) C_{n}^{i}(l)\right] \\
& = \begin{cases}2 L \cdot e^{j \varphi\left(a_{1}, a_{0}\right)}, & m=n \\
q \cdot e^{j \varphi\left(a_{1}, a_{0}\right)}, & m \neq n\end{cases}
\end{aligned}
$$

Or

$$
|R|=\left\{\begin{array}{ll}
2 L, & m=n \\
q, & m \neq n
\end{array} \text { and } \phi(R)=\varphi\left(a_{1}, a_{0}\right)\right.
$$

By choosing the maximum value $|R|_{\max }$ from all $|R|$ results, the order number $m$ (i.e., the 6 bits of $\left\{a_{7}, a_{6}, \cdots a_{2}\right\}$ ) can be determined. And by judging phase $\phi(R)$, the 2 bits of $\left\{a_{1}, a_{0}\right\}$ can be made certain.

\section{Simulation and Performance Analysis}

To reveal the performance of AOC scheme, computer simulation is made in conditions of: data rate $R_{b}=2 \mathrm{~Kb} / \mathrm{s}$, AOC sequence length $L=8$, co-correlation parameter $q \leq 4$, and carrier frequency $f_{0}=1 \mathrm{MHz}$. The bit error rate (BER) performance of AOC versus CCK [3] is shown in Figure 4.

Figure 4 shows that the proposed AOC scheme has $1.7 \mathrm{~dB}$ gain or so when BER is $10^{-4}$. This result is basically according with the conclusion given in the end of section 2 .

\section{Conclusions}

This paper presents a kind of spread spectrum scheme using approximate orthogonal complex (AOC) sequences. The aboratively selected AOC sequences have little co-correlation between any two sequences of AOC space, and the co-correlation parameter can be altered to enlarge or reduce the AOC space, so that the modulation efficiency can be changed. Performance analysis illustrates that the proposed AOC scheme has better processing gain than the existing CCK scheme. This is due to the smaller co-correlation than that of CCK sequences. The AOC scheme may find wide application in band-limited while requiring good transmission performance cases.

\section{References}

[1] A. J. Viterbi, "CDMA: Principles of spread spectrum communication," Prentice Hall, ISBN: 0201633744, April 1995.

[2] X. W. Zeng, et al., "Influences of PN code correlative characteristics on tamed spread spectrum," ACTA Electronica SINICA, Vol. 25, September 1997.

[3] C. Andren and M. Webster, "CCK modulation delivers 11Mbps for high rate IEEE 802.11 Extension,” Wireless symposium/portable by design conference, Spring 1999. 